\title{
Nelder-Mead Based Iterative Algorithm for Optimal Antenna Beam Patterns in Ad Hoc Networks
}

\author{
Vinay B. Ramakrishnaiah, Robert F. Kubichek, Suresh S. Muknahallipatna* \\ Department of Electrical \& Computer Engineering, University of Wyoming, Laramie, WY, USA \\ Email: *sureshm@uwyo.edu
}

How to cite this paper: Ramakrishnaiah, V.B., Kubichek, R.F. and Muknahallipatna, S.S. (2017) Nelder-Mead Based Iterative Algorithm for Optimal Antenna Beam Patterns in Ad Hoc Networks. Journal of Computer and Communications, 5, 117134.

https://doi.org/10.4236/jcc.2017.57012

Received: April 10, 2017

Accepted: May 20, 2017

Published: May 23, 2017

Copyright $\odot 2017$ by authors and Scientific Research Publishing Inc. This work is licensed under the Creative Commons Attribution International License (CC BY 4.0).

http://creativecommons.org/licenses/by/4.0/

cC) (i) Open Access

\begin{abstract}
Directional antennas shape transmission patterns to provide greater coverage distance and reduced coverage angle. Use of adaptive directional antenna arrays can minimize interference while also being more energy efficient. When used in an ad-hoc network, this reduces interference among transmitting nodes and thereby increases throughput. Such "smart antennas" use digital beamforming based on signal processing algorithms to compute the appropriate weights to form effective antenna patterns. Smart antennas require the knowledge of the signal received at each antenna in the antenna array, thereby increasing the complexity of hardware and cost. Also, conventional smart antennas optimize results for each individual node, while it is preferable to have a global optimal solution. A problem that has not been addressed is how to compute individual beam patterns that maximize some measure of global network performance. Historically, the focus has been on finding node antenna patterns that give locally optimal performance. In this paper, we investigate a low hardware complexity beamforming approach aimed at improving global performance that uses average Noise-to-Signal ratio as the performance measure. Given a multi-hop route from source to destination, beam patterns are shaped to maximize average signal-to-noise ratio across all nodes on the route, which reduces bit-error rates and extends battery and network lifetime. The antenna weights are sequentially adjusted across all nodes in the route to achieve optimization across the network. By using phase-only weights, hardware costs are minimized. The performance of the algorithm using different path loss models is explored.
\end{abstract}

\section{Keywords}

Phased Array, Adaptive Antennas, Beamforming, Ad Hoc Networks, Network-Wide Optimization, Low Hardware Complexity, Global

Performance Improvement 


\section{Introduction}

Ad hoc networks are wireless networks capable of autonomous communication independent of pre-established infrastructure. Energy efficiency is an important consideration as it determines node and network lifetime. Usually, communication takes place using omni-directional antennas that radiate signals in all directions. This not only wastes transmission power at the node but also acts as a source of interference to other nodes.

Power efficiency can be improved using smart antennas to direct the beam in the desired direction while minimizing gain in interference directions. Smart antennas use digital beamforming (DBF) methods, which require separate transceiver chains, A/D and D/A converters, and DSPs for each antenna in the array (Figure 1(a)). Given access to each antenna signal, DBF can adaptively manipulate antenna weights to maximize SNR in real time. The downside is that DBF is unsuitable when low cost and complexity are required by the application. More importantly, such adaptive algorithms give locally optimal performance and don't typically consider global cost and performance constraints.

Analog beamforming (ABF) is a low complexity alternative to smart antennas. The system relies on a single transceiver and power splitter/combiner (Figure 1(b)). Computer controlled analog amplifiers/attenuators and phase shifters are used to form the desired beam patterns. The disadvantage of ABF is that the computer only has access to the combined received signals. Furthermore, bidirectional amplifiers or step attenuators add cost and complexity to the system.

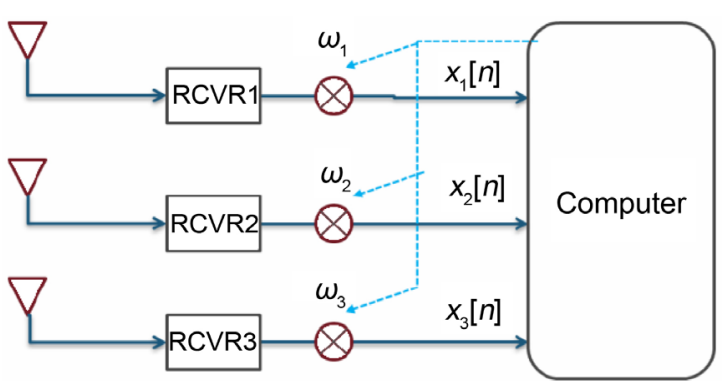

(a)

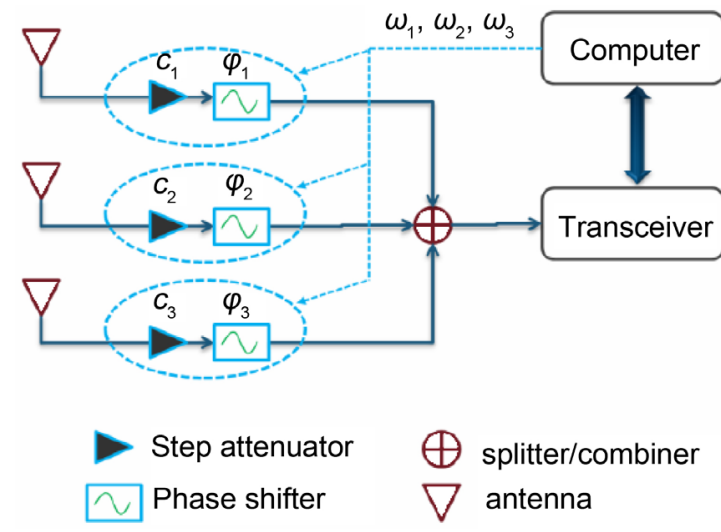

(b)

Figure 1. (a) DBF architecture (Image source: [24]). (b) $\mathrm{ABF}$ architecture (Image source: [24]). 
Therefore, the research described here focuses on techniques that use phase-only weights.

Since there is little established previous research on network-optimized antenna beamforming [1], we initially focus on fixed non-mobile networks such as wireless mesh sensor networks where node locations are known a-priori.

For a given route, we assume $\mathrm{N}$ nodes are active for relaying packets across a known route, and each node has an $\mathrm{M}$-antenna array of omnidirectional antennas. All other nodes in the network are considered to be interference sources. The SNR at each node can be predicted using simple path loss models (such as the two-ray model or the Walfisch-Ikegami model [2]), along with known positions of all nodes to estimate signal and interference powers.

We use SNR averaged over all network nodes as the measure of network performance. This is straightforward to compute and directly relates to network performance metrics such as bit error rate and battery life. To accomplish the optimization, we first find weights $w_{j k}, k=1,2, \cdots, M$ for each node $j$ that minimize average network noise-to-signal ratio (NSR). Once all $N$ node weight vectors are known $\left(w_{j k}, j=1,2, \cdots, N\right)$ weights are recomputed iteratively until the average NSR reaches a stable minimum. This provides a beamforming solution that intents to improve the performance of the network globally.

This approach can be simplified since each node in the route only communicates with previous and next hop neighbors and all other nodes are treated as interference. Thus, "average NSR" is taken to be the arithmetic mean over all the nodes along the route. Initially, only one route is considered; network nodes that are not in the current route are assumed to be interference nodes and their beam patterns remain omnidirectional or unchanged if assigned a pattern previously.

This paper is organized as follows: In Section 2, we discuss the related work on adaptive beamforming in ad hoc networks. Section 3 provides background theory on phased array antennas and the optimization technique used to obtain optimized beampattern in this paper. Section 4 explains the beamforming algorithm to provide improved global solution that increases the overall average Signal-to-Noise ratio (SNR) of the network. Section 5 outlines a brief discussion of noise in antenna systems. The simulation and analysis of results of the proposed beamforming algorithm is presented in Section 6. Section 7 concludes this paper with a discussion on performance issues and future work to be done on the developed algorithm.

\section{Related Work}

The problem of finding a global optimal solution to minimize the power consumption or maximize the Signal-to-Noise ratio is challenging in ad hoc networks. Previous researchers [3] [4] [5] [6] have proposed sub-optimal solutions using co-operative beamforming where nodes in an ad hoc network co-operate to act as antenna arrays. The authors of [3] formulate an optimization problem in a generalized scenario with multiple primary and secondary receivers that maximizes the weighted sum transmission rate of secondary destinations while 
maintaining the asynchronous interference at the primary receivers below their target thresholds. One of the main limitations of this approach is the huge amount of feedback overhead involved in the co-operative formation control algorithms [6] [7] [8]. There are other researches that propose non-cooperative beamforming [1] [9] [10], which includes selfish nodes that do not co-operate with other communicating nodes and use smart antenna approaches for adaptive beamforming. The problem of finding a global solution is challenging mainly because of the lack of natural ordering of the actions in ad hoc networks [9]. Zeydan et al. [9] point out that simple changes like variations in the power of one node pair affect the Signal-to-Interference-plus-noise ratio (SINR) of other node pairs and vice versa.

Thornburg et al. [11] assess the performance of millimeter wave (mmWave) devices to reduce interference due to directional antennas and building blockages. They formulate the performance of mmWave ad hoc networks in a stochastic geometry framework under the assumption of adaptive directional beamforming implementation and simulate beampatterns using a sectored model [12]. The sectored model represents beam patterns under the assumption that the antenna array can provide enough degrees of freedom to form the purported beam but can result in errors or sub-optimal solutions if the antenna array is unable to produce the desired beam.

The authors of [13] study the impact of using directional antennas and beamforming schemes on the connectivity of cognitive radio ad hoc networks. They evaluate the performance using randomized beamforming and center directed beamforming in ad hoc networks. However, they do not employ any adaptive beamforming techniques to adapt to changes in the network.

Anbaran et al. have proposed a method using smart antennas that delivers beamforming performance close to that of phased array antennas without having any constraints on the antenna spacing, and compare it to the conventional Electrically Steerable Passive Array Radiator (ESPAR) [13] system. An Electrically Steerable Passive Array Radiator (ESPAR) antenna delivers a low-cost solution for analog adaptive beamforming. The ESPAR antenna consists of one center element connected to the source and several surrounding parasitic elements reactively terminated to ground. The beam pattern can be controlled by adjusting the value of the reactance that terminates the parasitic elements. This method is efficient but results in relatively larger beam width and higher side lobe levels. However, smart antennas require information about signal from each antenna in the array, which increases the hardware cost. The authors in [14] focus on using smart antennas in ad hoc networks and they also provide simulation results with a seven element ESPAR antenna using QualNet.

The Kalman filter [15] based adaptive array processing is fast and efficient but requires transceivers and additional circuitry at each antenna in the node, which adds to cost and complexity of the circuit. Our approach uses a single transceiver and phase only weights to reduce the hardware complexity and cost. The major objective of [16] is to study the overall efficiency of an ad hoc network in terms 
of the antenna pattern and the length of the training sequence used by the beamforming algorithms. They conclude from the simulation results that the radiation patterns with smaller beam widths and lower side-lobes result in higher network capacity. Reference [17] describes an approach that makes use of a directional antenna to improve the performance of multicasting in ad hoc wireless networks. The antenna beam width at the network nodes is determined in such a way that both the node's transmit power and the interference among simultaneous transmissions are reduced while the signal power at the intended receivers remains unchanged.

In this paper, we provide a low-hardware complexity phased array antenna beamforming technique that provides a network-wide optimized solution to deliver a global improvement in performance. We assume that the optimal route from source to destination is known a-priori, and that it can be obtained from any convenient routing protocol. All the antenna weights are calculated centrally (not in a distributed fashion at individual nodes) to minimize the signal-to-interference-plus-noise ratio (SINR) at each node. This makes our approach a separate layer that is independent of the routing protocol used and can be added on top of existing networks. Similar to cooperative techniques, beamforming is done to take into account terrain and node locations, but our method does not require internode communication and the associated overheads. Like smart antennas, we adjust beams to adapt to local terrain and other node signals, but antenna weights are computed off-line and prior to network setup, with periodic updates made as needed. Smart antennas perform local optimization, while our method seeks to optimize globally across the network. Also, hardware complexity of the proposed system is much lower than smart antenna-based radios.

\section{Background Theory}

\subsection{Phase Only Weights}

The transmitted signal can be represented as $r(t)=2 s(t) \cos \left(\omega_{0} t\right)=s(t) \mathrm{e}^{j \omega_{0} t}+s(t) \mathrm{e}^{-j \omega_{0} t}$, where $\omega_{0}=2 \pi f_{0}=$ angular frequency. The block diagram of the receiver is represented in Figure 2. The mixer output signal $u(t)=s(t) \mathrm{e}^{-2 j \omega_{0} t}+s(t) \mathrm{e}^{0}$, but the high frequency component is removed by the low pass filter giving $w(t)=s(t)$. So, we can safely assume the transmitted signal to be $r(t)=s(t) \mathrm{e}^{j \omega_{0} t}$ to avoid unnecessary calculations.

A uniform linear array consisting of $M$ antenna elements is shown in Figure 3. Consider a single instant of time $t$, giving a snapshot of the wavefront for a signal arriving from direction $\theta$. By looking at Figure 3, it is evident that wave

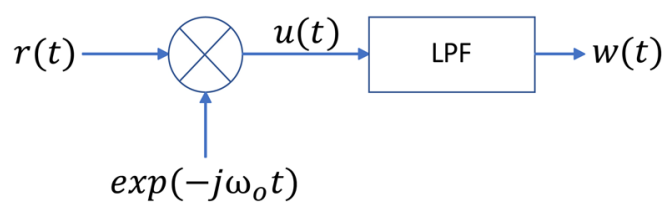

Figure 2. Block diagram of the receiver. 

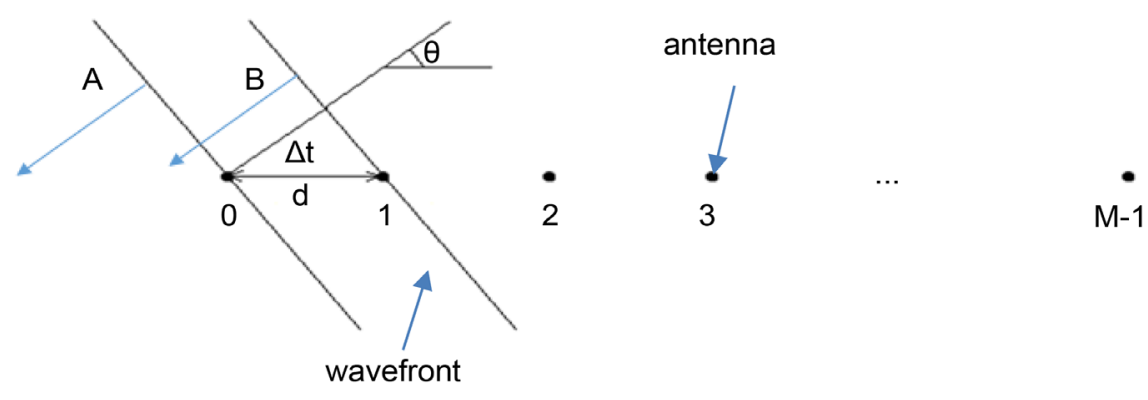

Figure 3. Uniform linear array of $\mathrm{M}$ elements.

B touches antenna \# 1 before it touches antenna \#0. Let the wavefront at antenna $\# 0$ be $s_{0}(t)=s(t) \mathrm{e}^{j \omega_{0} t}$, then the wavefront at the antenna \#1 is $s_{1}(t)=s_{0}(t+\Delta t)$. We assume a "low-pass narrow-band" signal $\mathrm{s}(t)$ with Bandwidth $\ll f_{0}$. Therefore $s(t+\Delta t) \approx s(t)$, where $\Delta t=\frac{d \cos \theta}{c}$ and $c=\lambda f_{0}$. Therefore $\Delta t=\frac{2 \pi d \cos \theta}{\omega_{0} \lambda}$. The signal at the receiver for antenna $\mathrm{m}$ is given as

$$
r_{m}(t) \simeq s(t) \mathrm{e}^{j \omega_{0}(t+m \Delta t)}=s(t) \mathrm{e}^{j \omega_{0}\left(t+\frac{2 \pi m d \cos \theta}{\omega_{0} \lambda}\right)} .
$$

For the entire array, the received signal will be

$r(t)=s_{k}(t) \mathrm{e}^{j \omega_{0} t}\left[1, \mathrm{e}^{j k d \cos \theta}, \mathrm{e}^{j 2 k d \cos \theta}, \cdots\right]^{\mathrm{T}}$, where $k=\frac{2 \pi}{\lambda}=$ wavenumber.

The received signal is represented as $r(t)=2 s(t) \cos \left(\omega_{0} t\right)+v(t)$, where $s(t)$ is a narrow band message signal and $v(t)$ is white noise. The receiver down-converts the signal resulting in a complex base-band signal $y(t)$. For example, the result for a uniform linear array is:

$$
\begin{gathered}
y(t)=\left[\begin{array}{c}
1 \\
\mathrm{e}^{j k d \cos \theta} \\
\vdots \\
\mathrm{e}^{j(M-1) k d \cos \theta}
\end{array}\right] s(t)+\left[\begin{array}{c}
v_{0}(t) \\
\vdots \\
v_{M-1}(t)
\end{array}\right] \\
y(t)=h(\theta) s(t)+V,
\end{gathered}
$$

where $k, d$, and $\theta$ are the wave number, antenna element separation distance, and direction of arrival (DOA), respectively, and $h(\theta)$ is called the steering vector. Note that $h(\theta)$ must be modified for each specific antenna array geometry to give proper delay characteristics in the direction $\theta$. Now, it is possible to find a linear filter $K$ that minimizes the effects of noise without distorting the signal.

$$
\hat{s}=\sum k_{i} y_{i}=K^{*} y .
$$

The filter output is unbiased as shown by

$$
E[\hat{s}]=E\left[K^{*} y\right]=E\left[K^{*} h(\theta) s+K^{*} V\right]=K^{*} h(\theta) s=s .
$$

The gain in direction $\theta$ is:

$$
G(\theta)=w^{\mathrm{T}} h(\theta),
$$


where $w$ is the weight vector which applies to antenna elements and depends on the optimization method.

There are several ways to find the weight vector $w$. For example, it is possible to find $w$ that minimizes output noise power while holding $G\left(\theta_{s}\right)=1$ in signal direction $\theta_{s}$. This is called a "Minimum Variance Distortion-less Response (MVDR)" filter [18]. This method is effective but requires knowledge of the noise plus interference covariance matrix, which requires access to individual antenna signals and is not possible using analog beam forming. In addition, MVDR weight magnitudes are unconstrained and thus not suitable as phaseonly weight vectors.

For this research, we use the Nelder-Mead (NM) search algorithm to find phase-only weights minimizing a desired fitness function. NM is one of the most widely used methods for nonlinear unconstrained optimization. For example, to generate the optimal gain pattern $G_{\text {opt }}(\theta)$, we enforce the phase-only constraint by fixing the weights as $w_{k}=\mathrm{e}^{j \phi_{k}}$ and then use NM to search the error surface $|e(\phi)|^{2}=\sum_{\theta}\left|G_{o p t}(\theta)-G(\theta, \phi)\right|^{2}$ for a minimum. Here, $G(\theta, \phi)$ is the gain pattern generated by phase-only weights $w=\mathrm{e}^{j \phi}$, where $\phi=\left[\phi_{1}, \phi_{2}, \ldots, \phi_{M}\right]^{\mathrm{T}}$. However, in most cases the optimal gain pattern $G_{\text {opt }}(\theta)$ is not known a-priori so we use NSR as the fitness function. As this directly relates to global network performance and the resulting gain pattern $G\left(\theta, \phi_{\operatorname{minNSR}}\right)$ is expected to be a reasonable approximation of $G_{\text {opt }}(\theta)$.

\subsection{Nelder-Mead Algorithm}

The Nelder-Mead (NM) algorithm [19] is one of the most widely used methods for non-linear unconstrained optimization. The Nelder-Mead method attempts to minimize a scalar valued non-linear function of $n$ real variables using only the function values without any derivative information. This algorithm uses a simplex of $\mathrm{n}$-dimensional vectors $x$. Let $x_{i}$ denote the list of points in the current simplex, $i=1, \cdots, n+1$. Because we seek to minimize the function $f, x_{1}$ is referred to as the best point, and $x_{n+1}$ as the worst point. Four scalar parameters reflection $(\rho)$, expansion $(\chi)$, contraction $(\gamma)$, and shrinkage $(\sigma)$ are specified for Nelder-Mead method.

The following indicates one iteration of the Nelder-Mead algorithm [20]:

- The $\mathrm{n}+1$ vertices are ordered such that $f\left(x_{1}\right) \leq f\left(x_{2}\right) \leq \cdots \leq f\left(x_{n+1}\right)$.

- The reflection point, $x_{r}$, is computed as $x_{r}=\bar{x}+\rho\left(\bar{x}-x_{n+1}\right)=(1+\rho) \bar{x}-\rho x_{n+1}$, where $\bar{x}=\sum_{i=1}^{n} \frac{x_{i}}{n}$. Evaluate

- $f_{r}=f\left(x_{r}\right)$. If the value $f_{1} \leq f_{r}<f_{n}$ the reflected point $\mathrm{x}_{\mathrm{r}}$ is accepted and the iteration terminates.

- The expansion point is computed as $x_{e}=\bar{x}+\chi\left(x_{r}-\bar{x}\right)=\bar{x}+\rho \chi\left(\bar{x}-x_{n+1}\right)=(1+\rho \chi) \bar{x}-\rho \chi x_{n+1}$ if $f_{r}<f_{1}$ and the value of the function $f_{e}$ at $x_{e}$ is evaluated. The iteration is terminated after retaining either $x_{e}\left(f_{e}<f_{r}\right)$ or $x_{r}\left(f_{e}>f_{r}\right)$.

- Contraction is performed by computing the contracted point 
$x_{c}=\bar{x}+\gamma\left(x_{r}-\bar{x}\right)$. A new simplex is obtained by using the contracted point

$x_{c}$ if it is better than the worst point.

- The function is evaluated by replacing all the points by $v_{i}=x_{1}+\sigma\left(x_{i}-x_{1}\right)$, $i=2, \cdots, n+1$, except for the best point. The new vertices $x_{1}, v_{2}, \cdots, v_{n+1}$ are used for update in the next iteration.

Before discussing performance of NM solutions applied to an entire network, we first focus on individual antenna array performance by comparing NM-based array solutions to the popular ESPAR antenna array described earlier. We modeled an antenna array with 7 antennas arranged in a circular geometry that is similar to the ESPAR antenna used by [14] for simulation as shown in Figure 4. In contrast to the ESPAR antenna, our antenna array has all elements connected to the source and the phases are individually adjusted to control the beam pattern. Both antenna arrays were designed to maximize the output SINR under the desired and interfering signals, which requires maximum gain in the signal direction and minimum gain in all others. Comparison of the beam patterns (Figure 5) with simulation results from [14] show that the NM-designed array has a much narrower beam width and lower side-lobe levels than the ESPAR antenna.

\section{Algorithm}

In this section, we describe a technique for finding network-optimized beam patterns (and the associated complex phase-only antenna weights) for all nodes along a route. This is a joint solution, where individual beam patterns depend on the antenna patterns of adjacent nodes. The approach is iterative, such that the iteration proceeds in a sequential manner from node to node along the route. The average NSR of the nodes along the route is calculated at each iteration and NM is used to minimize the average NSR by trying different values of antennas weights. The scheme is repeated until convergence.

The algorithm is summarized in the following steps:

- Given all node locations, compute the distance $d_{i j}$ and the angle $\theta_{i j}$ between the nodes $i$ and $j$.

- For a given source and destination node, use a routing protocol to determine which nodes are members of the route. Nodes not included in the route are treated as interference sources.

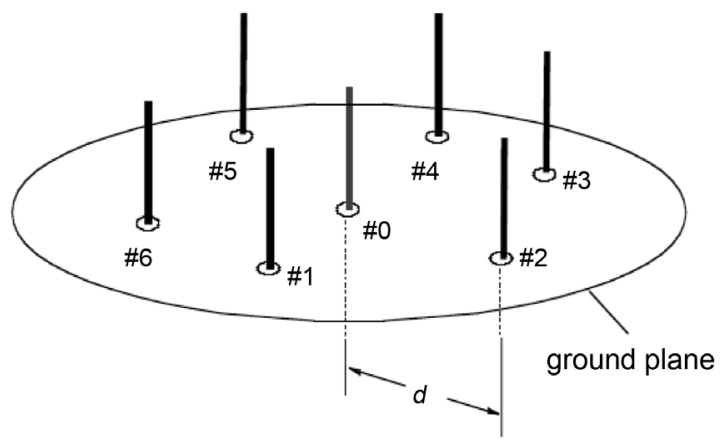

Figure 4. The ESPAR antenna (Image source: [13]). 


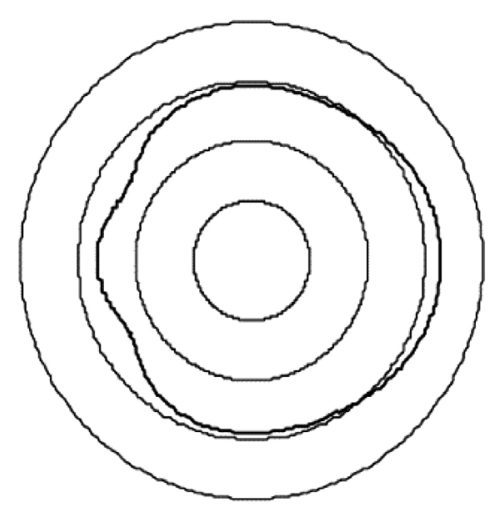

(a)

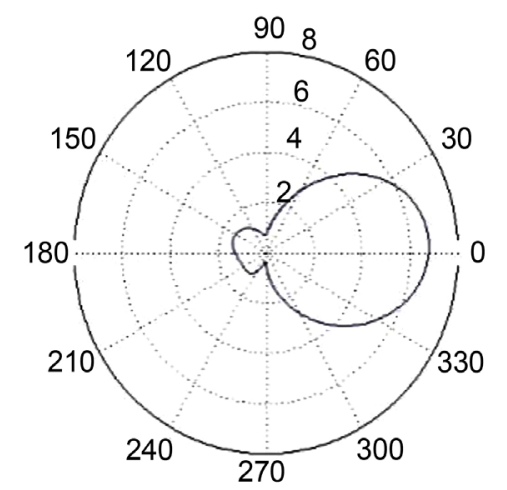

(c)

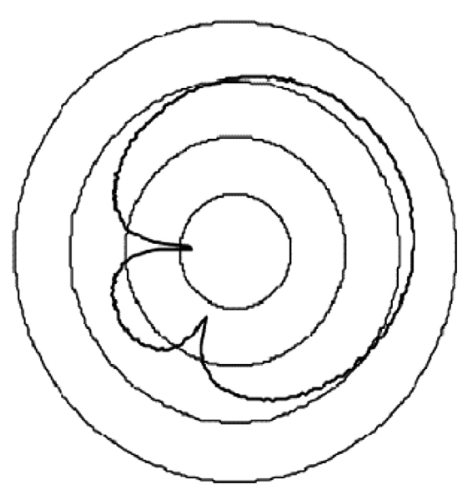

(b)

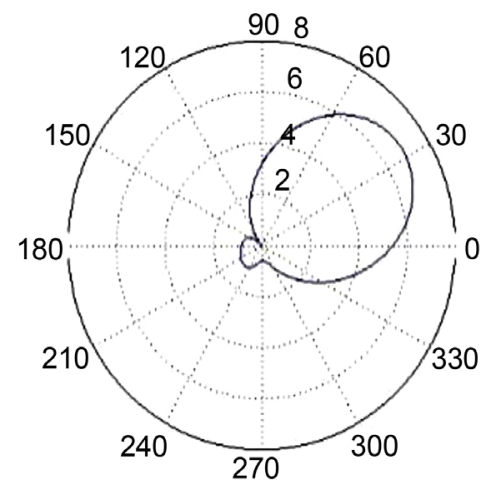

(d)

Figure 5. Directional beam patterns obtained by simulation (a) \& (b) ESPAR pattern at $0^{\circ}$ and $30^{\circ}$ (Image source: [14]). (c) \& (d) NM optimized pattern at $0^{\circ}$ and $30^{\circ}$.

- Calculate path loss between the transmitting and receiving nodes using a suitable path loss model.

- Compute received power at node $i, P_{R i j}$ due to signal source $j$ with transmit power $P_{T p}$ where $i$ and $j$ represent nodes on the route. Then compute the total received signal power at node $i$ using $P_{R i}^{S}=\sum_{j} P_{R i j}$. Antenna gains are calculated using Equation (3).

- Similarly, total interference power at node $i$ can be calculated using $P_{R i}^{I}=\sum_{j} P_{R i j}$ where $P_{R i j}$ represents received power from interference node $j$, i.e., node $j$ is not a node on the route.

- Ambient noise can be included by computing a suitable noise temperature and using it to calculate the noise power $N_{i}$.

- Assume an initial weight vector for the antennas at each node to compute an initial gain $G_{i}(\theta)$; using this, calculate the received signal power, interference power, and the noise power. From these values calculate $N S R$ at each of the $M$ nodes in the route as well as the route-average NSR denoted by $\overline{N S R}$ :

- $\quad N S R_{i}=\frac{P_{R i}^{I}+N_{i}}{P_{R i}^{S}}$

- $\overline{N S R}=\frac{1}{M} \sum_{i=1}^{M} N S R_{i}$. 
- Apply NM to compute the weight vector at each node using $\overline{N S R}$ as the fitness function.

- Using the obtained weight vector for the $i$-th node, calculate the gain in the direction of the $j$-th node $G_{i}\left(\theta_{i j}\right)=w_{i}^{\mathrm{T}} h\left(\theta_{j}\right)$, where $w_{i}=\mathrm{e}^{j \varphi_{i}}$ is the weight vector at node $i$ defined by antenna phase vector $\varphi_{i}^{\mathrm{T}}=\left[\varphi_{i 1}, \varphi_{i 2}, \cdots\right]$. NM minimizes NSR by trying different values of $\varphi_{i}$ to get the weights. Note that each candidate weight vector affects the beam pattern of the current node, thereby changing the node's NSR as well as the average $\overline{N S R}$ for the route. The iteration proceeds in a sequence along the nodes in the route and is repeated until convergence. Each time the weight vector at a node is calculated, it considers the refined weight vector of its neighbors from the previous iteration. Convergence is reached when there is no longer significant reduction in $\overline{N S R}$ in a complete pass of the algorithm through the route.

Each node along the route must communicate with its previous and next hop neighbors. In each pass, the algorithm tries to refine the weight vector at each node, such that the average noise-to-signal ratio is minimized. As the algorithm tries to reduce the average NSR by minimizing the individual terms of the summation $\sum_{i=1}^{M} N S R_{i}$, it always tries to improve the SNR at each node. Also, as the iteration proceeds in a sequence, all the nodes in the route are equally favored.

\section{Noise in Antenna Systems}

Along with the desired and interference signals from various sources, the antenna system also receives noise from radiating sources of natural origin. These sources include cosmic noise, noise from the sun, noise from the ground, etc. Apart from these noises, the receiving system and amplifiers used with antennas also contribute to the system noise. Usually, the noise power received by an antenna is represented using antenna noise temperature and the noise from different sources can be combined in an additive manner.

The antenna noise temperature is the temperature of an equivalent fictitious resistor that would give rise to the same noise per unit bandwidth as that of the antenna output at a given frequency. The received noise power per unit bandwidth is given by $S_{a}=k T_{a}$, where $k$ is the Boltzmann's constant and $T_{a}$ is the noise temperature of the antenna and is computed as

$$
T_{a}=\frac{1}{4 \pi} \iint G(\theta, \phi) T(\theta, \phi) \mathrm{d} \theta \mathrm{d} \phi,
$$

where $G(\theta, \phi)$ is the antenna gain and $T(\theta, \phi)$ is the sky brightness. Their product is integrated over the entire solid angle to compute the antenna noise temperature. There are empirical formulae available to calculate different factors that contribute to the sky brightness. For example, [21] have provided a formula for approximating the cosmic noise temperature, which is given by

$$
T_{c(\text { average })}=290 \lambda^{2}=\frac{2.6 \times 10^{7}}{f^{2}}
$$

where $T_{c}$ is the absolute temperature (in Kelvin), $\lambda$ is the wavelength in me- 
ters and $f$ is the frequency in MHz. The thermal noise in the receiving system will also have a noise temperature in addition to the noise from natural sources. The amplifier not only adds noise but also amplifies the noise at the input by a factor of the amplifier gain. Other factors like noise due to lossy elements also contribute to the system noise temperature. In general, the system noise temperature can be computed as

$$
T_{\text {sys }}=T_{a}+T_{\text {rec }}+T_{\text {amp }}+T_{\text {feed }}+\cdots .
$$

Therefore, the noise power received by a receiver of bandwidth $B$ would be $k T_{\text {sys }} B$.

The system noise temperatures of a typical directive antenna vary between 40 $\mathrm{K}$ to $3000 \mathrm{~K}$ depending on the frequency of operation and [22] provide a graph of the noise temperatures of directive antenna for usual environment conditions that serves as an interim standard for most performance calculations. In our simulations, we have the operating frequency as $900 \mathrm{MHz}$ and the system noise temperature at this frequency is about $60 \mathrm{~K}$ at a beam elevation angle of $90^{\circ}$ from the zenith. The noise power of the antenna system is calculated as the product of the system noise temperature ( magnitude of order 2 at $900 \mathrm{MHz}$ ), Boltzmann's constant (magnitude of order -23 ), and the receiver bandwidth. To do an absolute worst case analysis, we assume a low pass filter in our system, so the bandwidth of our receiving system will be equal to $900 \mathrm{MHz}$ (magnitude of order 8 ). In reality, a receiver would use a bandpass filter, and we could reasonably assume a bandwidth of no more than $30 \mathrm{MHz}$. Therefore, the noise power in our system is approximately of the order of -12 . Considering a scenario of an ad hoc network with typical node separation of $1 \mathrm{~km}$, a transmitter power of $10 \mathrm{~W}$, and using the Friis equation of radio propagation to calculate the received power by an omni-directional antenna results in a magnitude of order -9 . This shows that noise power would be at least 3 orders of magnitude less than the interference power received in an ad hoc network. Hence, we ignore the noise power in our computation of optimal weights for the antennas.

\section{Simulation and Analysis}

The simulations were performed using MatlabR2015a [24] under the assumption of a known hop sequence from source to destination. Figure 6 shows an example route through a network comprising seven total nodes and hop sequence 1-2-3-4. Each node in the hop sequence is shown along with its final computed linear gain pattern. Nodes 5, 6, and 7 represent interfering nodes whose positions were chosen randomly. The operating frequency was chosen to be $900 \mathrm{MHz}$. Each node has a square grid antenna array containing nine antennas with $\lambda / 4$ grid spacing. The transmitter power was fixed to be equal to $10 \mathrm{~W}$.

Figure 6 shows that the gain pattern for each node provides maximal gain toward both the previous and next node in the hop sequence. For example, node 1 tries to point the beam towards node 2, avoiding interference from nodes $5 \&$ 6. Figure 7 shows average network NSR converging quickly from $8 \mathrm{~dB}$ to $-22 \mathrm{~dB}$ after about six iterations providing an improvement in average NSR of $30 \mathrm{~dB}$. 


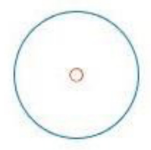

5

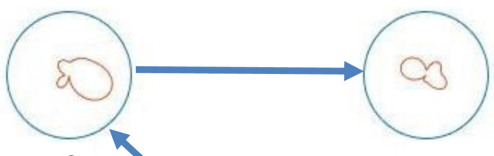

3

4

Route

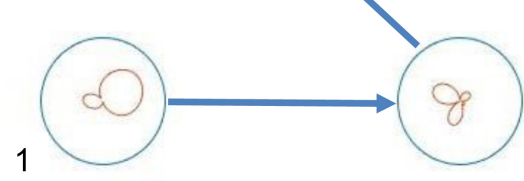

2

6

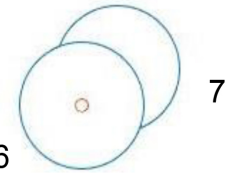

Figure 6. The beam patterns for route given by node sequence $1-2-3-4$.

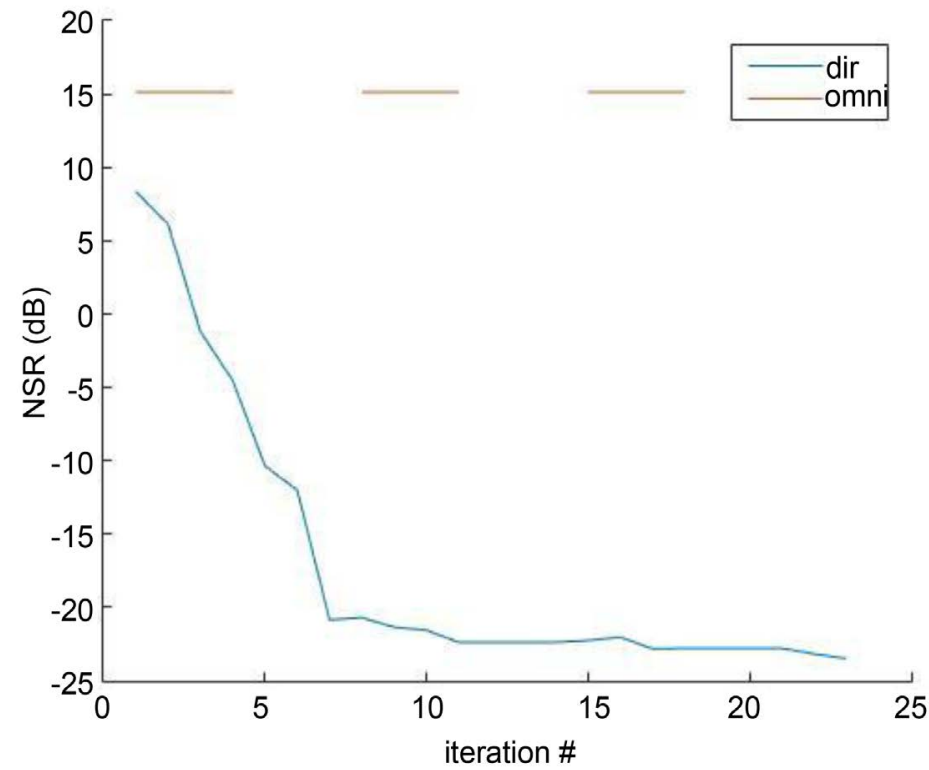

Figure 7. $\overline{N S R}$ vs. iteration number, showing convergence.

This shows that the proposed beamforming algorithm iteratively reduces the overall average NSR (therefore increases SNR) of the network, thereby, providing a globally improved solution by capturing all the changes in the network. The average NSR of the network using only omni-directional antennas is also shown in Figure 7 for comparison. To see if there is an improvement in performance, the SNR at each desired node of the network before and after optimizing the antenna weights is shown in Figure 8, which shows an improvement in SNR at each node of approximately $26 \mathrm{~dB}$. We also show the minimum transmitter power required at each node to maintain a SNR of $10 \mathrm{~dB}$ and found a significant reduction in total transmitter power as compared to using omnidirectional antenna, which can be seen in Figure 9. 


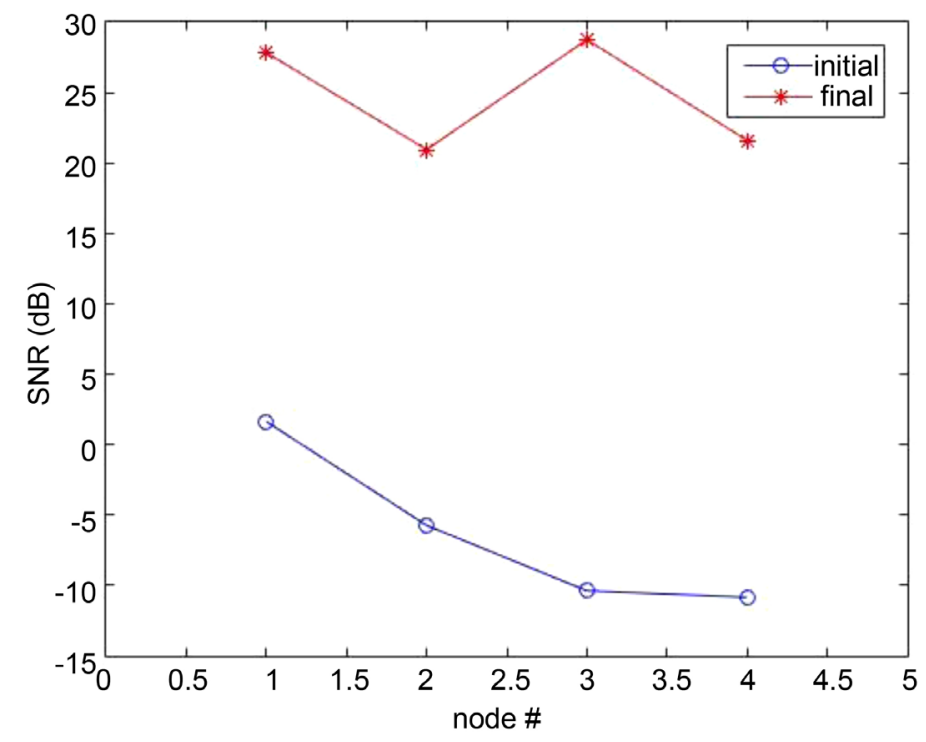

Figure 8 . The initial and final SNR at each desired node $(1-4)$ in the network.

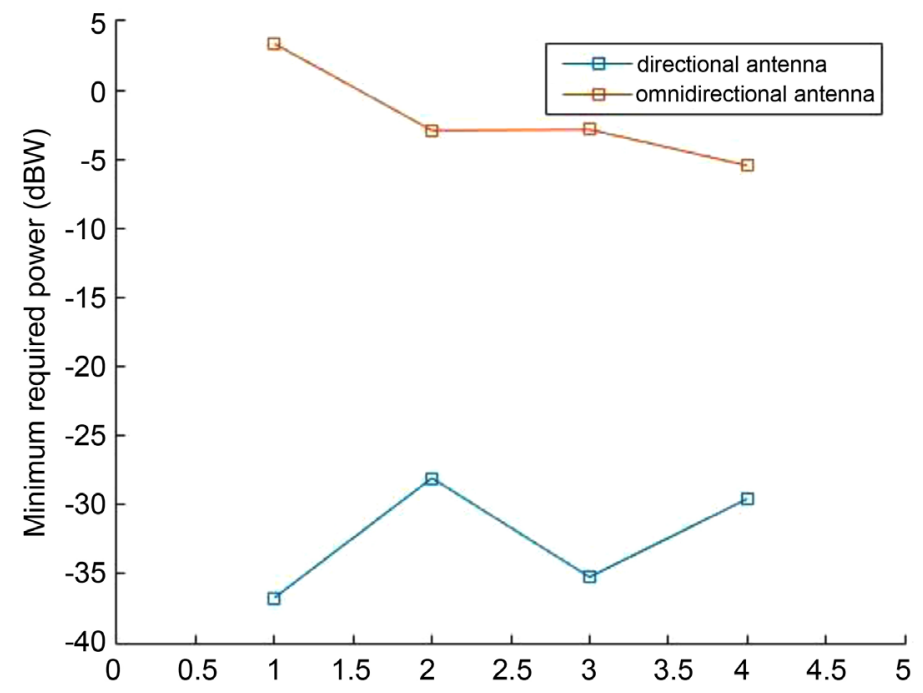

Figure 9. Comparison of the minimum power required at each node using omni-directional antennas and Nelder-Mead optimized arrays.

Figure 10 shows the histogram in decibel scale of average SNR for the considered topology over 1000 trials for two cases: 1) only omni-directional antennas are used, and 2) optimized antenna arrays are used. For each trial, different random interference locations were used. The average SNR for the directional case is considerably greater (about $35 \mathrm{~dB}$ ) than that for the omnidirectional case.

Similarly, we fixed the SNR to be $10 \mathrm{~dB}$ (approximate lower limit for acceptable bit error rates) and calculated the transmitter power required at each node to maintain that SNR. Figure 11 shows the plot of the total transmitter power required for 1000 different random instances of interference node positions. It is evident from the plot that the directional antenna arrays outperform the omnidirectional ones and minimize the total power consumption in the network. We 


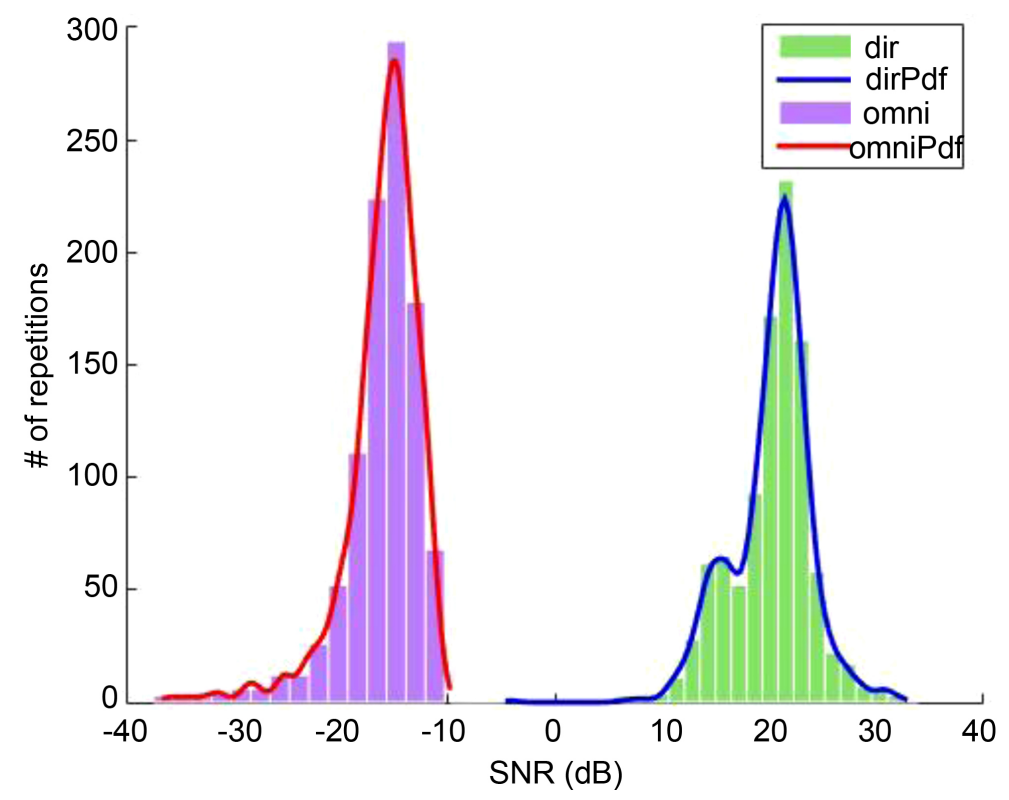

Figure 10. Average SNR using fixed transmitter power of $10 \mathrm{~W}$, for both directional and omni-directional cases for 1000 random trials.

present results from experiments performed using the free-space propagation model and the Walfish-Ikegami model (WIM) [23]. In WIM, we used an urban area with an average building height of $10 \mathrm{~m}$ with a building separation of $14 \mathrm{~m}$, assuming the streets to be $8 \mathrm{~m}$ wide. WIM is validated for base station heights ranging from $4-50 \mathrm{~m}$ along with the receiving antenna heights ranging from 1 $3 \mathrm{~m}$. In the case of ad hoc networks, as the same antenna system is connected to a transceiver we assumed the antenna height to be $3.5 \mathrm{~m}$ in our simulations. Comparing Figure 11(a) \& Figure 11(b), we can see that the total transmitter power required by the nodes using directional antennas using WIM to calculate the path loss is less when compared to using the FSM. This sounds counter-intuitive as the path loss calculated by WIM is always greater than or equal to the path loss calculated using FSM, resulting in higher received signal strength in case of FSM. This is not only true for the desired nodes but also for the interfering nodes (larger in number), thereby, decreasing the SNR. That is why we need more transmitter power to maintain a certain SNR while using FSM to calculate path losses.

In this approach, the improvement in performance is due to directional gain as well as nulling of interference. The gain pattern for the same topology as in Figure 6 is shown in Figure 12. Consider the communication between node 1 and 2. Node 1 has a directional gain of $16 \mathrm{~dB}$ towards node $2\left(0^{\circ}\right)$ and node 2 has a gain of about $11 \mathrm{~dB}$ in the direction towards node $1\left(180^{\circ}\right)$ providing a combined gain of $27 \mathrm{~dB}$. The data markers corresponding to the example angular locations are shown as black squares in Figure 12. As an example of interference nulling, the closest interference in the topology is at the bottom of node 1 $\left(\sim 270^{\circ}\right)$ and the algorithm places a null with the least gain in that direction. This improves the signal-to-noise ratio of communicating nodes significantly and is 


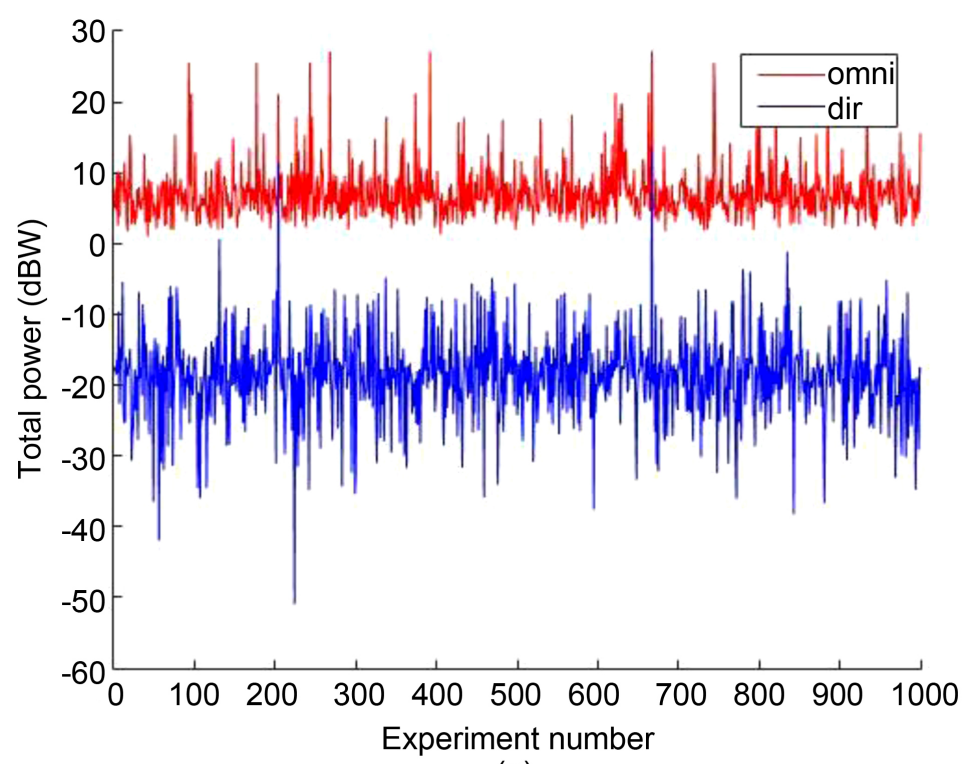

(a)

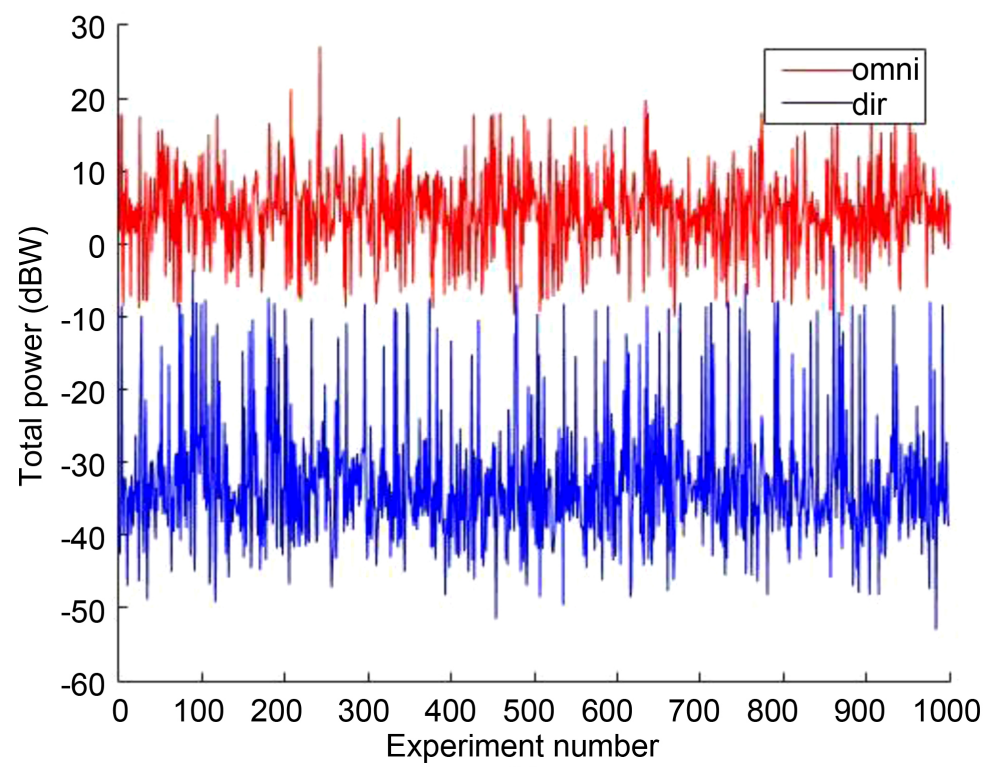

(b)

Figure 11. Total network transmitter power required to maintain a signalto-noise ratio of $10 \mathrm{~dB}$ at every node for 1000 random trials using (a) freespace model (FSM) (b) Walfisch-Ikegami model (WIM).

validated by the plot shown in Figure 10.

\section{Conclusion}

A low cost, low-complexity, and energy efficient solution for adaptive beam forming in ad hoc networks was proposed to increase the overall average SNR of the network. The approach uses the Nelder-Mead simplex method of unconstrained optimization to find antenna weights that provide a global solution for optimal beam patterns for a given network topology. This can provide lower bit error rate, increase throughput, and extend network life. The proposed method does not require transceivers and additional circuitry for each antenna in 


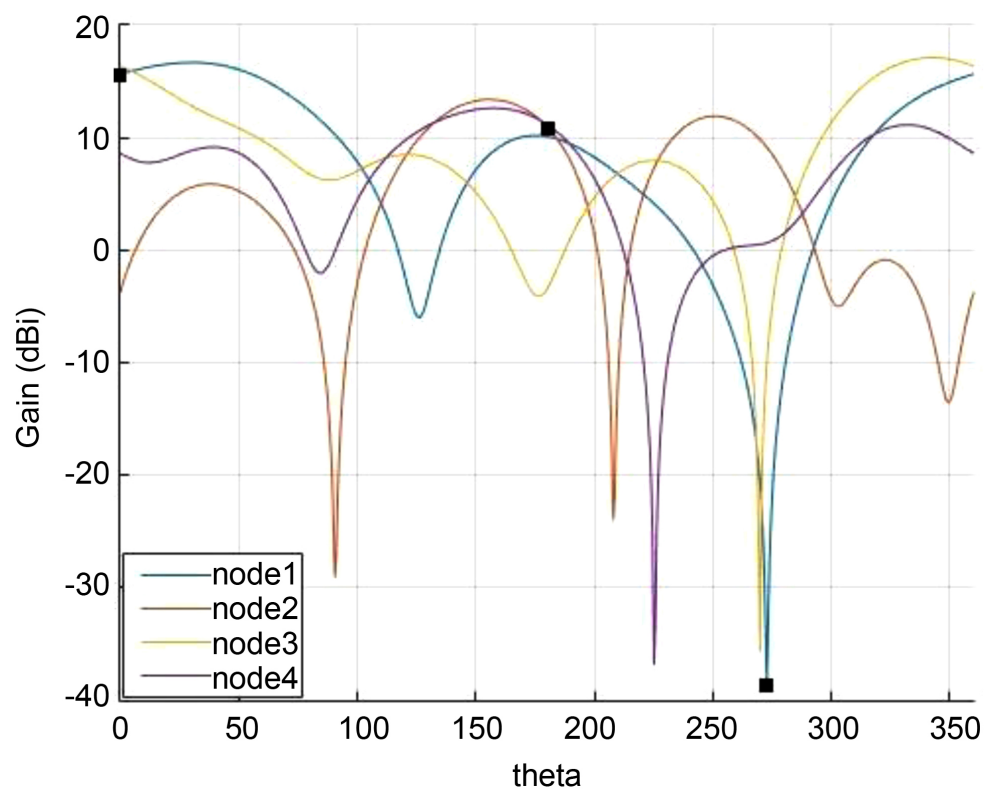

Figure 12. Beam pattern of the network nodes plotted on a linear scale. The data markers indicate the gain in the communicating directions and also the null in the direction of interference.

the node's antenna array as is the case for most smart antenna approaches. We have provided simulation results using the free-space model and the WalfischIkegami model of radio propagation. Both these models show similar results of increased SNR (about $35 \mathrm{~dB}$ improvement using a $3 \times 3$ antenna array) and decreased transmission power (decrease of $25-40 \mathrm{dBW}$ ) for the Nelder-Mead optimized arrays.

One potential problem with the current implementation is that suboptimal solutions may occur when the algorithm settles for a local minimum. Ways to reduce this problem such as randomly visiting nodes during the iteration are being investigated. Another potential issue is that average NSR may not be the best fitness function to use since it can be affected by a few high or low NSR outlier values along the route. Alternative fitness function to be considered might include total route transmission energy, network lifetime, or average network throughput. Currently, the proposed algorithm is suitable only for stationary networks like wireless sensor networks. This is mainly because considerable processing time is required to compute new beam patterns for every node in the network, which would be required each time the network changes. For example, a network with 3 desired nodes and an interference node takes approximately 3.5 seconds to converge on an Intel Xeon Sandy Bridge CPU ( $2 \mathrm{GHz})$. The use of high performance computing and neural networks can potentially improve the convergence speed and make the beamforming algorithm suitable for mobile ad hoc networks.

\section{References}

[1] Ramakrishnaiah, V.B., Kubichek, R.F. and Muknahallipatna, S.S. (2015) Optimiza- 
tion of Antenna Beam Pattern in Ad Hoc Networks for Optimal Global Performance. 58 th International Midwest Symposium on Circuits and Systems, Fort Collins, 2-5 August 2015, 1-4. https://doi.org/10.1109/mwscas.2015.7282046

[2] Haslett, C. (2008) Essentials of Radio Wave Propagation. Cambridge University Press, Cambridge.

[3] Hasan, M.H., Hossain, M. and Bhargava, V.K. (2016) Cooperative Beam-Forming for Cognitive Radio Based Broadcasting Systems in Presence of Asynchronous Interference. IEEE Transactions on Vehicular Technology, 66, 2311-2323. https://doi.org/10.1109/TVT.2016.2575059

[4] Arvola, A., Tolli, A. and Gesbert, D. (2016) A User Cooperative Beamforming Approach to PAPR Reduction in MIMO-OFDM Uplink. 50th Asilomar Conference on Signals, Systems and Computers, Asilomar, 6-9 November 2016. https://doi.org/10.1109/acssc.2016.7869133

[5] Feng, Y., Yang, Z., Zhu, W.-P., Li, Q. and Lv, B. (2017) Robust Cooperative Secure Beamforming for Simultaneous Wireless Information and Power Transfer in Amplify-and-Forward Relay Networks. IEEE Transactions on Vehicular Technology, 66, 2354-2366. https://doi.org/10.1109/TVT.2016.2578313

[6] Shamailzadeh, M. and Bastami, A.H. (2016) Cooperative Beamforming in Cognitive Radio Network with Network-Coded Bidirectional Filter-and-Forward Relaying. 24th Iranian Conference on Electrical Engineering, Shiraz, 10-12 May 2016, 14541459. https://doi.org/10.1109/iraniancee.2016.7585750

[7] Turki, I., Kammoun, I. and Siala, M. (2016) Cooperative Beamforming in Multi-Cells Multi-Users Networks with Quantized Feedback. International Symposium on Networks, Computers and Communications, Yasmine Hammamet, 11-13 May 2016, 1-6.

[8] Tharranetharan, S. and Hossain, J. (2016) Data Rate Fairness Cooperative Beamforming Techniques for Cognitive Radio Systems in the Presence of Asynchronous Interferences. IEEE Transactions on Communications, 64, 4083-4096. https://doi.org/10.1109/TCOMM.2016.2581805

[9] Zeydan, E., Kivanc-Tureli, D. and Tureli, U. (2010) Iterative Beamforming and Power Control for MIMO Ad Hoc Networks. Global Telecommunications Conference, Miami, 6-10 December 2010, 1-5.

[10] Choi, J. (2015) Interference-Leakage Based Non-Cooperative Beamforming with Low-Dimensional Approximation. IET Communications, 9, 1060-1067. https://doi.org/10.1049/iet-com.2014.0760

[11] Thornburg, A., Bai, T. and Heath, R.W. (2016) Performance Analysis of Outdoor mm Wave Ad Hoc Networks. IEEE Transactions on Signal Processing, 64, 40654079. https://doi.org/10.1109/TSP.2016.2551690

[12] Hunter, A.M., Andrews, J.G. and Weber, S. (2008) Transmission Capacity of Ad Hoc Networks with Spatial Diversity. IEEE Transactions on Wireless Communications, 7, 5058-5071. https://doi.org/10.1109/T-WC.2008.071047

[13] Kawakami, H. and Ohira, T. (2005) Electrically Steerable Passive Array Radiator (ESPAR) Antennas. Antennas and Propagation Magazine, 43-50.

[14] Bandyopadhyay, S., Roy, S. and Ueda, T. (2010) Enhancing the Performance of Ad Hoc Wireless Networks with Smart Antennas. CRC Press, Boca Raton.

[15] Hu, J. and Zhong, H. (1998) Kalman Filter for Adaptive Antennas. Wuhan University Journal of Natural Sciences, 3, 187-191. https://doi.org/10.1007/BF02827549

[16] Bellofiore, S., Foutz, J., Govindarajula, R., Bahçeci, I., Balanis, C.A., Spanias, A.S., 
Capone, J.M. and Duman, T.M. (2002) Smart Antenna System Analysis, Integration and Performance for Mobile Ad-Hoc Networks (MANETs). IEEE Transactions, 50, 571-581. https://doi.org/10.1109/TAP.2002.1011222

[17] Wang, K., Chiasserini, C.F., Proakis, J.G. and Rao, R.R. (2002) RISE: Reducing Interference and Saving Energy through Multicasting in Ad Hoc Wireless Networks. MILCOM, 7-10 October 2002, 826-832.

[18] Vorobyov, S.A., Chen, H. and Gershman, A.B. (2008) On the Relationship between Robust Minimum Variance Beamformers with Probabilistic and Worst-Case Distortionless Response Constraints. IEEE Transactions on Signal Processing, 56, 5719-5724. https://doi.org/10.1109/TSP.2008.929866

[19] Nelder, J.A. and Mead, R. (1965) A Simplex Method for Function Minimization. The Computer Journal, 7, 308-313. https://doi.org/10.1093/comjnl/7.4.308

[20] Lagarias, J.C., Reeds, J.A., Wright, M.H. and Wright, P.E. (1998) Convergence Properties of the Nelder-Mead Simplex Method in Low Dimensions. SIAM Journal on Optimization, 9, 112-147. https://doi.org/10.1137/S1052623496303470

[21] Hogg, D.C. and Mumford, W.W. (1960) The Effective Noise Temperature of the Sky. The Microwave Journal, 3, 80-84.

[22] Blake, L.V. (1961) Antenna and Receiving-System Noise-Temperature Calculation. U.S. Naval Research Laboratory, Washington DC.

[23] Walfisch, J. and Bertoni, H.L. (1988) A Theoretical Model of UHF Propagation in Urban Environments. IEEE Transactions on Antennas and Propagation, 36, 1788 1796. https://doi.org/10.1109/8.14401

[24] Bhamidipati, S.Y.S. (2012) Low Complexity Hybrid Digital/Analog Phased Array for Ad Hoc Networks. University of Wyoming, Laramie. https://doi.org/10.1109/icara.2015.7081204

Submit or recommend next manuscript to SCIRP and we will provide best service for you:

Accepting pre-submission inquiries through Email, Facebook, LinkedIn, Twitter, etc. A wide selection of journals (inclusive of 9 subjects, more than 200 journals)

Providing 24-hour high-quality service

User-friendly online submission system

Fair and swift peer-review system

Efficient typesetting and proofreading procedure

Display of the result of downloads and visits, as well as the number of cited articles

Maximum dissemination of your research work

Submit your manuscript at: http://papersubmission.scirp.org/

Or contact jcc@scirp.org 